\title{
Sb Grain Boundary Segregation in Rapidly Solidified Cu-Sb Alloy
}

\author{
Chunfei Li*, Masashi Watanabe*, John Li**, David W. Ackland* and David B. Williams* \\ *Material Science and Engineering Department, Lehigh University, Bethlehem, PA 18015 \\ **Semiconductor Research \& Development Center, IBM Microelectronics, Semiconductor \\ Research \& Development Center, Hopewell Jct., NY 12533
}

The segregation of trace elements to grain boundary in alloys is often undesirable because of the resulted embrittlement. There are two possible approaches to prevent embrittling segregation: (a) adding suitable extra elements; (b) quenching an alloy from the melt or from very high temperature. While many attempts to add extra elements have been made, there are few reports concerning the prevention of segregation by rapid quenching. With respect to the method of segregation characterization, recently, Analytical Electron Microscopy (AEM) plays an important role. AEM is capable of characterizing both the type of grain boundary and analyzing the composition of the same area. Previous AEM studies have indicated that the segregation depends on the type (misorientation and plane) of the grain boundaries. Because of the variety of grain boundary types, statistically significant studies are required to draw firm conclusions about the relationship between boundary type and chemistry. The introduction of a Vacuum Generators HB603 dedicated scanning transmission electron microscope (STEM) equipped with a Energy Dispersive X-ray Spectrometer (EDS) permits such grain boundary compositional analysis to be performed efficiently. As for the characterization of grain boundary type, the recent development of the Automatic Crystallography for Transmission Electron Microscopy (ACT) provides the possibility of characterizing grain boundary type automatically [1]. The present paper investigates the effect of rapid quenching on the grain boundary segregation and report the first attempt to combine ACT with AEM in a segregation study. $\mathrm{A} \mathrm{Cu}_{0.98} \mathrm{Sb}_{0.02}$ (in weight percent) alloy was chosen for the present study because $\mathrm{Sb}$ grain boundary segregation and the resultant embrittlement are well documented [2].

A cylindrical alloy of $3 \mathrm{~mm}$ diameter was prepared by casting the melt in a $\mathrm{Cu}$ mold. The cooling rate of this method is estimated to be approximately $100 \mathrm{~K} / \mathrm{s}$. To prepare the specimen for TEM observation, a slice from the alloy was mechanically polished, dimpled and finally ion milled. The characterization of the grain boundary type was performed by using a Philips EM 420 Transmission Electron Microscope (TEM) equipped with the ACT capability. The segregation of Sb on the same grain boundaries was analyzed by using HB 603 STEM.

Several grain boundaries were characterized in the present study. Fig. 1 shows a typical result of ACT characterization of a grain boundary. The middle picture is the bright field image of an area containing two grains. The diffraction patterns corresponding to the two grains are shown on the left and right sides, respectively. These were reconstructed from the ACT results. From the diffraction patterns, it is calculated that the relative orientation of the two grains is formed through a $45^{\circ}$ rotation along the $<-7-111>$ crystalline direction. In term of the Coincidence Site Lattice, this implies a $\Sigma$ value larger than 43 for the grain boundary. Fig. 2 shows the bright field and $\mathrm{Sb}$ elemental mapping images of the same area. From the mapping image, it is clear that Sb segregated to the grain boundary even in the present rapid quenching condition. In contrast to the above mentioned result, investigation on a small angle grain boundary found no Sb segregation. Continued studies aim to analyze many boundaries in the same specimen. 
References

[1] S.I. Wright et al, Mater. Sci. Forum 273-275 (1998) 209.

[2] K.S. Yu et al, Metal. Trans. 14A (1983) 2447.

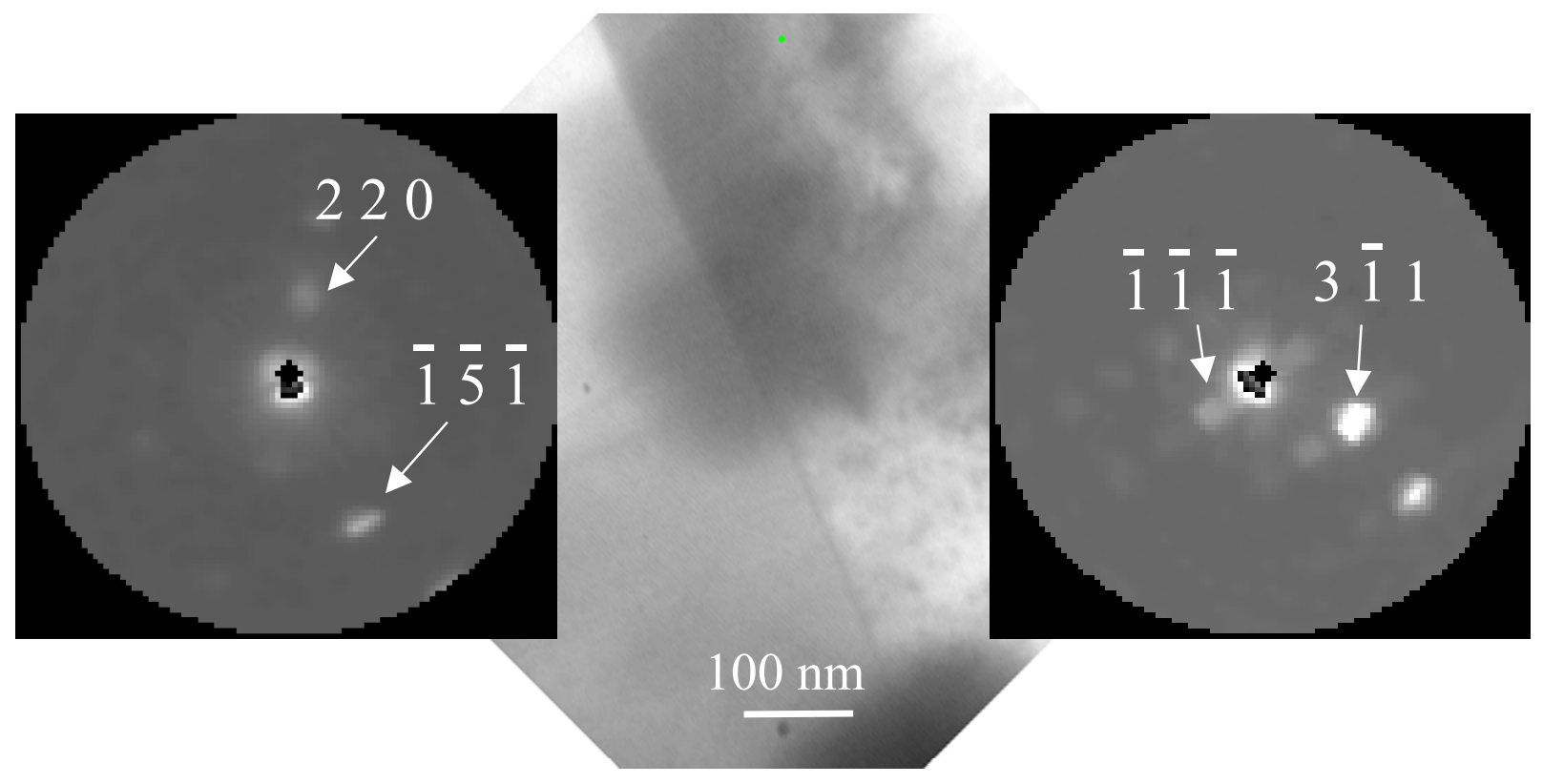

Fig. 1 An example of grain boundary characterization using Automatic Crystallography for TEM. The bright field TEM image and the reconstructed diffraction patterns were shown.
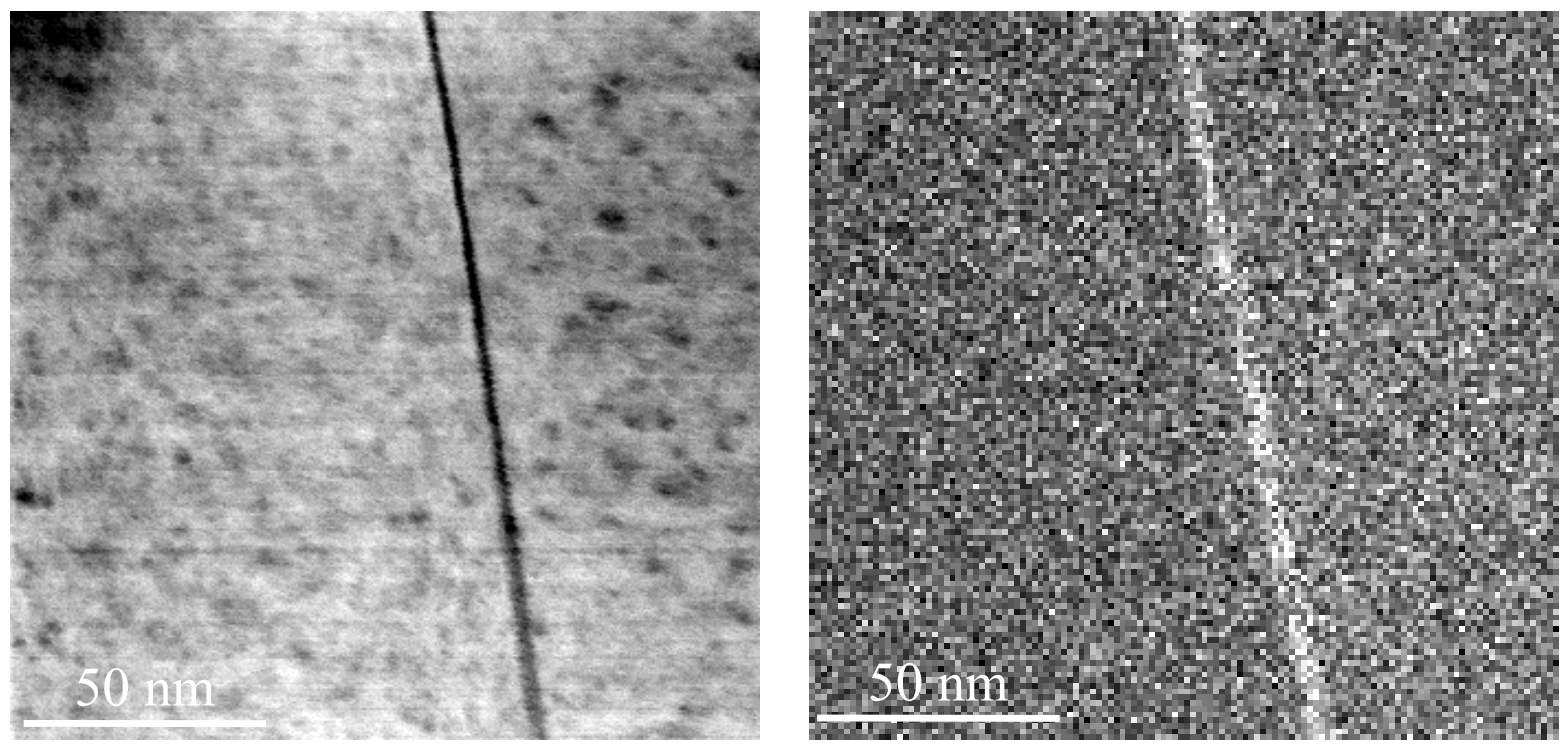

Fig.2 Bright field scanning transmission electron microscope image and Sb elemental map at the same grain boundary as that of Fig. 1. 